\title{
Cross-intersecting families of labeled sets
}

\author{
Huajun Zhang* \\ Department of Mathematics, Zhejiang Normal University, Jinhua 321004, P.R. China \\ huajunzhang@zjnu.cn
}

Submitted: Jan 30, 2012; Accepted: Jan 14, 2013; Published: Jan 21, 2013

Mathematics Subject Classifications: 05D05, 06A07

\begin{abstract}
For two positive integers $n$ and $p$, let $\mathcal{L}_{p}$ be the family of labeled $n$-sets given by$$
\mathcal{L}_{p}=\left\{\left\{\left(1, \ell_{1}\right),\left(2, \ell_{2}\right), \ldots,\left(n, \ell_{n}\right)\right\}: \ell_{i} \in[p], i=1,2 \ldots, n\right\} .
$$

Families $\mathcal{A}$ and $\mathcal{B}$ are said to be cross-intersecting if $A \cap B \neq \emptyset$ for all $A \in \mathcal{A}$ and $B \in \mathcal{B}$. In this paper, we will prove that for $p \geqslant 4$, if $\mathcal{A}$ and $\mathcal{B}$ are cross-intersecting subfamilies of $\mathcal{L}_{\mathfrak{p}}$, then $|\mathcal{A}||\mathcal{B}| \leqslant p^{2 n-2}$, and equality holds if and only if $\mathcal{A}$ and $\mathcal{B}$ are an identical largest intersecting subfamily of $\mathcal{L}_{p}$.
\end{abstract}

Keywords: EKR theorem; Intersecting family; cross-intersecting family; labeled set

\section{Introduction}

For a positive integer $n$, let $[n]$ denote the set $\{1,2, \ldots, n\}$. Given a set $X$, by $\left(\begin{array}{l}X \\ k\end{array}\right)$ we denote the set of all $k$-subsets of $X$, and let $2^{X}$ denote the set of all subsets of $X$. A family $\mathcal{A}$ of sets is said to be $t$-intersecting if $|A \cap B| \geqslant t$ for every pair $A, B \in \mathcal{A}$. Usually, $\mathcal{A}$ is called intersecting if $t=1$.

The Erdös-Ko-Rado Theorem [15] says that if $\mathcal{A}$ is an intersecting subfamily of $\left(\begin{array}{c}{[n]} \\ k\end{array}\right)$ where $n \geqslant 2 k$, then $|\mathcal{A}| \leqslant\left(\begin{array}{l}n-1 \\ k-1\end{array}\right)$. This theorem is a central result in extremal set theory and inspires abundant fruits in this field, for an excellent introduction to this we recommend the survey paper [13].

This theorem has many generalizations, analogs and variations. First, finite sets are analogous to finite vector spaces $([17,18,20])$, permutations $([11,12,27])$ and labeled sets (signed sets $[4,6]$ or colored sets [22]), etc. Second, the intersection condition was

\footnotetext{
*The author is supported by the National Natural Science Foundation of China (No.11001249 and No.11171310) and the Natural Science Foundation of Zhejiang Province (No.LY12A01006.)
} 
generalized to $t$-intersection and cross-intersection. Here, families $\mathcal{A}_{1}, \mathcal{A}_{2}, \ldots, \mathcal{A}_{m}$ are said to be cross-intersecting if $A \cap B \neq \emptyset$ for any $A \in \mathcal{A}_{i}$ and $B \in \mathcal{A}_{j}, i \neq j$. Many authors studied the bound of $\sum_{i=1}^{m}\left|\mathcal{A}_{i}\right|([19,5,6,7,8,9,10,29,30])$, and Pyber [25] first considered the bound of $|\mathcal{A}||\mathcal{B}|$ for cross-intersecting families $\mathcal{A}$ and $\mathcal{B}$. His result was slightly refined by Matsumoto and Tokushige [24] and Bey [3] as follows.

Theorem 1. If $\mathcal{A} \subseteq\left(\begin{array}{c}{[n]} \\ k\end{array}\right)$ and $\mathcal{B} \subseteq\left(\begin{array}{c}{[n]} \\ \ell\end{array}\right)$ are cross-intersecting with $n \geqslant \max \{2 k, 2 \ell\}$, then

$$
|\mathcal{A}||\mathcal{B}| \leqslant\left(\begin{array}{l}
n-1 \\
k-1
\end{array}\right)\left(\begin{array}{l}
n-1 \\
\ell-1
\end{array}\right)
$$

Moreover, the equality holds if and only if $\mathcal{A}=\left\{A \in\left(\begin{array}{c}{[n]} \\ k\end{array}\right): i \in A\right\}$ and $\mathcal{B}=\left\{B \in\left(\begin{array}{c}{[n]} \\ \ell\end{array}\right)\right.$ : $i \in B\}$ for some $i \in[n]$, unless $n=2 k=2 \ell$.

Tokushige [26] and Ellis, Friedgut and Pilpel [14] generalized the above result to cross$t$-intersecting families of finite sets and cross-t-intersecting subfamilies of the symmetric group $S_{n}$, respectively. This paper provides an analogue of Theorem 1 for families whose sets we refer to as labeled sets, following [5].

For an $n$-tuple $\mathfrak{p}=\left(p_{1}, p_{2}, \ldots, p_{n}\right)$ such that $p_{1}, p_{2}, \ldots, p_{n}$ are positive integers with $p_{1} \leqslant p_{2} \leqslant \cdots \leqslant p_{n}$, we define the family $\mathcal{L}_{\mathfrak{p}}$ of labeled sets by

$$
\mathcal{L}_{\mathfrak{p}}=\left\{\left\{\left(1, \ell_{1}\right),\left(2, \ell_{2}\right), \ldots,\left(n, \ell_{n}\right)\right\}: \ell_{i} \in\left[p_{i}\right], i=1,2 \ldots, n\right\} .
$$

Berge [2] determined the maximum size of intersecting families of labeled $n$-sets, Livingston [23] characterized partial optimal intersecting families and Borg [5] completely solved it by using the shift operator in an inductive argument.

Theorem 2 (Berge, Livingston, Borg). If $\mathcal{A}$ is an intersecting subfamily of $\mathcal{L}_{\mathfrak{p}}$, then $|\mathcal{A}| \leqslant$ $p_{2} p_{3} \cdots p_{n}$. When $p_{1} \geqslant 3$, equality holds if and only if $\mathcal{A}=\left\{\left\{\left(1, \ell_{1}\right),\left(2, \ell_{2}\right), \ldots,\left(n, \ell_{n}\right)\right\}\right.$ : $\left.\ell_{i}=j\right\}$, where $p_{i}=p_{1}$ and $j \in\left[p_{1}\right]$.

In [5], Borg also determined the upper bound of $\sum_{1 \leqslant i \leqslant m}\left|\mathcal{A}_{i}\right|$ for cross-intersecting subfamilies $\mathcal{A}_{1}, \mathcal{A}_{2}, \ldots, \mathcal{A}_{m}$ of $\mathcal{L}_{\mathfrak{p}}$.

In this paper, we consider a special case: $p_{1}=p_{2}=\cdots=p_{n}=p$. In this case, we write $\mathcal{L}_{\mathfrak{p}}$ as $\mathcal{L}_{p}$. The main result in this paper is the following theorem.

Theorem 3. Let $n$ and $p$ be two positive integers with $p \geqslant 4$. If $\mathcal{A}$ and $\mathcal{B}$ are crossintersecting families in $\mathcal{L}_{p}$, then

$$
|\mathcal{A}||\mathcal{B}| \leqslant p^{2 n-2}
$$

and equality holds if and only if $\mathcal{A}=\mathcal{B}=\left\{\left\{\left(1, \ell_{1}\right),\left(2, \ell_{2}\right), \ldots,\left(n, \ell_{n}\right)\right\}: \ell_{i}=j\right\}$ for some $i \in[n]$ and $j \in[p]$.

We will present some preliminary results in the next section, and complete the proof of the above theorem in Section 3. 


\section{Preliminary Results}

For the labeled set $\mathcal{L}_{p}$, we can construct a simple graph, whose vertex set is $\mathcal{L}_{p}$, and $A, B \in \mathcal{L}_{p}$ are adjacent if and only if $A \cap B=\emptyset$. For convenience, this graph is also denoted by $\mathcal{L}_{p}$. Set $\Gamma=S_{n} \imath S_{p}=\left\{\left(f, g_{1}, g_{2}, \ldots, g_{n}\right): f \in S_{n}\right.$ and $\left.g_{1}, g_{2}, \ldots, g_{n} \in S_{p}\right\}$, the wreath product of the symmetric groups on $[n]$ and $[p]$. For $\sigma=\left(f, g_{1}, g_{2}, \ldots, g_{n}\right) \in \Gamma$ and $\left\{\left(1, \ell_{1}\right),\left(2, \ell_{2}\right), \ldots,\left(n, \ell_{n}\right)\right\} \in \mathcal{L}_{p}$, define

$$
\sigma\left(\left\{\left(1, \ell_{1}\right), \ldots,\left(n, \ell_{n}\right)\right\}\right)=\left\{\left(f(1), g_{1}\left(\ell_{1}\right)\right), \ldots,\left(f(n), g_{n}\left(\ell_{n}\right)\right)\right\}
$$

Then $\Gamma$ acts transitively on $\mathcal{L}_{p}$. In other words, the graph $\mathcal{L}_{p}$ is vertex-transitive. Moreover, every intersecting subfamily of the labeled set $\mathcal{L}_{p}$ corresponds to an independent set of the graph $\mathcal{L}_{p}$. In the sequel we shall alternatively use the terms "set" and "graph" when referring to $\mathcal{L}_{p}$.

For a graph $G$, let $\alpha(G)$ denote the independence number of $G$. Given a subset $A$ of $V(G)$, we define

$$
\begin{gathered}
N_{G}(A)=\{b \in V(G):\{a, b\} \in E(G) \text { for some } a \in A\} \\
\bar{N}_{G}(A)=V(G)-N_{G}(A) .
\end{gathered}
$$

If $G$ is clear from the context, for simplicity, we will omit the index $G$. For $B \subseteq V(G)$, by $G[B]$ we denote the induced subgraph of $G$. For short, we abbreviate $\alpha(G[B])$ to $\alpha(B)$.

For the labeled set $\mathcal{L}_{p}$ we construct another graph $\widehat{\mathcal{L}}_{p}$, whose vertex set is the set $\left\{(A, B) \in \mathcal{L}_{p} \times \mathcal{L}_{p}: A \cap B \neq \emptyset\right\}$, and $\left(A_{1}, B_{1}\right)$ and $\left(A_{2}, B_{2}\right)$ are non-adjacent if and only if $A_{1} \cap B_{2} \neq \emptyset$ and $B_{1} \cap A_{2} \neq \emptyset$. By definition it is easy to see that if $\mathcal{A}$ and $\mathcal{B}$ are cross-intersecting subfamilies of $\mathcal{L}_{p}$, then $\mathcal{A} \times \mathcal{B}$ is an independent set of $\widehat{\mathcal{L}}_{p}$. Therefore, $|\mathcal{A}||\mathcal{B}| \leqslant \alpha\left(\widehat{\mathcal{L}}_{p}\right)$. To complete the proof of Theorem 3, it suffices to determine the size and structure of the maximum independent sets in $\widehat{\mathcal{L}}_{p}$.

Note that the action of $\Gamma$ on $\mathcal{L}_{p}$ induces an action on the graph $\widehat{\mathcal{L}}_{p}$ defined by $\sigma(A, B)=$ $(\sigma(A), \sigma(B))$ for $\sigma \in \Gamma$ and $(A, B) \in \widehat{\mathcal{L}}_{p}$. For $1 \leqslant i \leqslant n$, set $\widehat{\mathcal{L}}_{p, i}=\left\{(A, B) \in \mathcal{L}_{p} \times \mathcal{L}_{p}\right.$ : $|A \cap B|=i\}$. Clearly, $|A \cap B|=|\sigma(A) \cap \sigma(B)|$ holds for all $\sigma \in \Gamma$ and $A, B \in \mathcal{L}_{p}$, and it is easy to verify that $\widehat{\mathcal{L}}_{p, 1}, \widehat{\mathcal{L}}_{p, 2}, \ldots, \widehat{\mathcal{L}}_{p, n}$ are all orbits of $\Gamma$ on $\widehat{\mathcal{L}}_{p}$. In other words, every induced subgraph $\widehat{\mathcal{L}}_{p, i}$ is vertex-transitive.

In the context of vertex-transitive graphs, the following result named the "no-homomorphism lemma" is useful to get bounds on the size of independent sets.

Lemma 4 (Albertson and Collins [1]). Let $G$ and $G^{\prime}$ be two graphs such that $G$ is vertextransitive and there exists a homomorphism $\phi: G^{\prime} \mapsto G$. Then $\frac{\alpha(G)}{|V(G)|} \leqslant \frac{\alpha\left(G^{\prime}\right)}{\left|V\left(G^{\prime}\right)\right|}$, and the equality holds if and only if for any independent set $I$ of cardinality $\alpha(G)$ in $G, \phi^{-1}(I)$ is an independent set of cardinality $\alpha\left(G^{\prime}\right)$ in $G^{\prime}$.

The following Lemma is a variation of the above. 
Lemma 5. (see [11, Theorem 3]) Let $G$ be a vertex-transitive graph, and $\Omega$ a transitive subgroup of $A u t(G)$. Let $I$ be an independent set of $G$, and let $B \subseteq V(G)$, then $\frac{|I|}{|V(G)|} \leqslant$ $\frac{\alpha(B)}{|B|}$. Equality holds if and only if $|I \cap \sigma(B)|=\alpha(B)$ holds for all $\sigma \in \Omega$.

Proof. Set $\mathcal{D}=\{\sigma(B): \sigma \in \Omega\}$ and $\mathcal{D}_{u}=\{D \in \mathcal{D}: u \in D\}$ for $u \in V(G)$. Note that the action of $\Omega$ on $V(G)$ is transitive. The size of $\mathcal{D}_{u}$, denoted by $r$, is independent of the choice of $u$. Hence, $r|V(G)|=|B||\mathcal{D}|$. On the other hand, for each $D \in \mathcal{D}, I \cap D$ is also an independent set of $D$, and so $|D \cap I| \leqslant \alpha(G[B])$. Therefore, $r|I| \leqslant \alpha(G[B])|\mathcal{D}|$. Combining the above two inequalities gives $\frac{|I|}{|V(G)|} \leqslant \frac{\alpha(G[B])}{|B|}$, and equality holds if and only if $|D \cap I|=\alpha(G[B])$ for each $D \in \mathcal{D}$.

Since all $\widehat{\mathcal{L}}_{p, i}$ are vertex-transitive, the above lemma can be applied to them. In more detail, let $\widehat{\mathcal{K}}$ be a subset of $\widehat{\mathcal{L}}_{p}$ such that $\widehat{\mathcal{K}} \cap \widehat{\mathcal{L}}_{p, i} \neq \emptyset$ for $1 \leqslant i \leqslant n$. Write $\widehat{\mathcal{K}}_{i}=\widehat{\mathcal{K}} \cap \widehat{\mathcal{L}}_{p, i}$ for $i \in[n]$. Then, for any independent set $\widehat{\mathcal{I}}$ of $\widehat{\mathcal{L}}_{p}$ and $i \in[n],\left|\widehat{\mathcal{I}} \cap \widehat{\mathcal{L}}_{p, i}\right| \leqslant \alpha\left(\widehat{\mathcal{L}}_{p, i}\right)$, and by Lemma $5, \alpha\left(\widehat{\mathcal{L}}_{p, i}\right) \leqslant\left|\widehat{\mathcal{L}}_{p, i}\right| \frac{\alpha\left(\widehat{\mathcal{K}}_{i}\right)}{\left|\hat{\mathcal{K}}_{i}\right|}$. Therefore,

$$
|\widehat{\mathcal{I}}|=\sum_{i=1}^{n}\left|\widehat{\mathcal{I}} \cap \widehat{\mathcal{L}}_{p, i}\right| \leqslant \sum_{i=1}^{k}\left|\widehat{\mathcal{L}}_{p, i}\right| \frac{\alpha\left(\widehat{\mathcal{K}}_{i}\right)}{\left|\widehat{\mathcal{K}}_{i}\right|},
$$

and equality holds if and only if $\left|\widehat{\mathcal{I}} \cap \widehat{\mathcal{L}}_{p, i}\right|=\alpha\left(\widehat{\mathcal{L}}_{p, i}\right)$ and $\left|\widehat{\mathcal{I}} \cap \widehat{\mathcal{L}}_{p, i} \cap \sigma(\widehat{\mathcal{K}})\right|=\alpha\left(\widehat{\mathcal{K}}_{i}\right)$ for all $i=1,2, \ldots, n$ and $\sigma \in \Gamma$. Equivalently, for each $\sigma \in \Gamma$,

$$
|\widehat{\mathcal{I}} \cap \sigma(\widehat{\mathcal{K}})|=\sum_{i=1}^{n}\left|\sigma^{-1}(\widehat{\mathcal{I}}) \cap \widehat{\mathcal{K}} \cap \widehat{\mathcal{L}}_{p, i}\right|=\sum_{i=1}^{n} \alpha\left(\widehat{\mathcal{K}}_{i}\right)=\alpha(\widehat{\mathcal{K}}) .
$$

We state it as a lemma as follows.

Lemma 6. Let $\widehat{\mathcal{K}}$ be a subset of $\widehat{\mathcal{L}}_{p}$ such that $\widehat{\mathcal{K}}_{i} \neq \emptyset$ for $1 \leqslant i \leqslant n$, where $\widehat{\mathcal{K}}_{i}=\widehat{\mathcal{K}} \cap \widehat{\mathcal{L}}_{p, i}$. If $\widehat{\mathcal{I}}$ is an independent set of $\widehat{\mathcal{L}}_{p}$, then

$$
|\widehat{\mathcal{I}}| \leqslant \sum_{i=1}^{n}\left|\widehat{\mathcal{L}}_{p, i}\right| \frac{\alpha\left(\widehat{\mathcal{K}}_{i}\right)}{\left|\widehat{\mathcal{K}}_{i}\right|}
$$

and equality holds if and only if $|\widehat{\mathcal{I}} \cap \sigma(\widehat{\mathcal{K}})|=\sum_{i=1}^{n} \alpha\left(\widehat{\mathcal{K}}_{i}\right)=\alpha(\widehat{\mathcal{K}})$ for each $\sigma \in \Gamma$.

Arrange the elements

$$
(1,1),(2,1), \ldots,(n, 1),(1,2),(2,2), \ldots,(n, 2), \ldots,(1, p),(2, p), \ldots,(n, p)
$$

in a cycle. Let $R_{i}$ denote the $i$ th $n$-interval $\{(s, j),(s+1, j) \ldots,(n, j),(1, j+1), \ldots,(s-$ $1, j+1)\}$ of this cycle, where $i=n(j-1)+s$ with $1 \leqslant s \leqslant n$. Set $\mathcal{R}=\left\{R_{1}, R_{2}, \ldots, R_{n p}\right\}$ and $\widehat{\mathcal{R}}=\{(A, B) \in \mathcal{R} \times \mathcal{R}: A \cap B \neq \emptyset\}$. Then, $\widehat{\mathcal{R}} \subseteq \widehat{\mathcal{L}}_{p}$ and $\widehat{\mathcal{R}}_{i}=\widehat{\mathcal{R}} \cap \widehat{\mathcal{L}}_{p, i} \neq \emptyset$ for each $1 \leqslant i \leqslant n$. 
Clearly, $R_{i} \cap R_{j} \neq \emptyset$ if and only if $|i-j|<n$ or $|i+n p-j|<n$ for $R_{i}, R_{j} \in \mathcal{R}$, and the subgraph of $\mathcal{L}_{p}$ induced by $\mathcal{R}$, which will also be denoted by $\mathcal{R}$, is isomorphic to the well-known circular graph $\operatorname{Circ}(n, n p)$. Here, the graph $\operatorname{Circ}(n, n p)$ has the vertex set $[n p]$, and $i$ and $j$ are not adjacent if and only if $|i-j|<n$ or $|n p+i-j|<n$. Hence, $\alpha(\mathcal{R})=n$, and by the well-known result of Katona [21], the maximum independent sets of $\mathcal{R}$ are stars. In the following we will prove that $\widehat{\mathcal{R}}$ is the desired subset.

Let $\mathcal{A}$ and $\mathcal{B}$ be cross-intersecting subfamilies of $\mathcal{R}$. Then, it is obvious that $\mathcal{B} \subseteq$ $\bar{N}_{\mathcal{R}}(\mathcal{A})$. For every non-empty $A \subset V(\operatorname{Circ}(n, n p))(p \geqslant 3)$, we have proved that if $|A| \geqslant 2 n$, $\bar{N}(A)=\emptyset$; if $|A|<2 n,|\bar{N}(A)|+|A| \leqslant 2 n$, and equality holds if and only if $A=$ $\{i, i+1, \ldots, i+|A|-1\}$ for some $i$ (see [16, Lemma 3.1] or [28, Lemma 2.3]). Therefore, if $\mathcal{A}$ and $\mathcal{B}$ are both non-empty, then $|\mathcal{A}|+|\mathcal{B}| \leqslant|\mathcal{A}|+\left|\bar{N}_{\mathcal{R}}(\mathcal{A})\right| \leqslant 2 n$. Note that $|\mathcal{A}||\mathcal{B}|=0$ if one of $\mathcal{A}$ and $\mathcal{B}$ is empty. So we have that $|\mathcal{A}||\mathcal{B}| \leqslant|\mathcal{A}|(2 n-|\mathcal{A}|) \leqslant n^{2}$, and equality holds if and only if $\mathcal{A}$ and $\mathcal{B}$ are some identical maximum independent set of $\mathcal{R}$. Therefore, $\alpha(\widehat{\mathcal{R}})=n^{2}$. In the following, we give a stronger result.

Lemma 7. Suppose $p \geqslant 4$. Then

$$
\alpha(\widehat{\mathcal{R}})=n^{2}=\sum_{i=1}^{n} \alpha\left(\widehat{\mathcal{R}}_{i}\right),
$$

and $\widehat{\mathcal{I}}$ is a maximum independent set of $\widehat{\mathcal{R}}$ if and only if $\widehat{\mathcal{I}}=\mathcal{S} \times \mathcal{S}$ for some maximum independent set of $\mathcal{R}$.

Proof. For any subsets $\mathcal{A}, \mathcal{B}$ of $\mathcal{R}$ and $1 \leqslant i \leqslant n$, set $(\mathcal{A}, \mathcal{B})_{i}=\left|(\mathcal{A} \times \mathcal{B}) \cap \widehat{\mathcal{R}}_{i}\right|$. Let $\mathcal{S}$ be a fixed maximum independent set of $\mathcal{R}$ and write $(\mathcal{S}, \mathcal{S})_{i}=a_{i}$. Clearly, $a_{i}$ does not depend on the choice of $\mathcal{S}$, and $\alpha(\widehat{\mathcal{R}})=n^{2}=\sum_{1 \leqslant i \leqslant n} a_{i}$. To complete the proof, it suffices to prove that $\alpha\left(\widehat{\mathcal{R}}_{i}\right)=a_{i}$ for each $1 \leqslant i \leqslant n$. To do this, we only need to verify that for every independent set $\widehat{\mathcal{I}}$ of $\widehat{\mathcal{R}},\left|\widehat{\mathcal{I}} \cap \widehat{\mathcal{R}}_{i}\right| \leqslant a_{i}$ for $1 \leqslant i \leqslant n$.

Let $\widehat{\mathcal{I}}$ be an independent set of $\widehat{\mathcal{R}}$. Then there exists a pair of cross-intersecting subfamilies $\mathcal{C}$ and $\mathcal{D}$ of $\mathcal{R}$ such that $\widehat{\mathcal{I}} \subseteq \mathcal{C} \times \mathcal{D}$. Since $|\mathcal{C}|+|\mathcal{D}| \leqslant 2 n$, we may assume $|\mathcal{C}|=s \leqslant n$.

We first consider the simple case when $\mathcal{C}$ consists of consecutive elements of $\mathcal{R}$. Without loss of generality, assume $\mathcal{C}=\left\{R_{n}, R_{n+1}, \ldots, R_{n+s-1}\right\}$. For $1 \leqslant t \leqslant n$, set $\mathcal{C}_{t}=\left\{R_{n}, R_{n+1}, \ldots, R_{n+t-1}\right\}$. Then, $\mathcal{D} \subseteq \bar{N}\left(\mathcal{C}_{s}\right)$. For each $1 \leqslant t<n$ and $1 \leqslant i \leqslant n$, it is easy to verify that

$$
\begin{aligned}
\mathcal{C}_{t+1} \times \bar{N}\left(\mathcal{C}_{t+1}\right) & =\left[\mathcal{C}_{t} \times \bar{N}\left(\mathcal{C}_{t+1}\right)\right] \cup\left[\left\{R_{n+t}\right\} \times \bar{N}\left(\mathcal{C}_{t+1}\right)\right] \\
& =\left[\mathcal{C}_{t} \times \bar{N}\left(\mathcal{C}_{t}\right)\right] \cup\left[\left\{R_{n+t}\right\} \times \bar{N}\left(\mathcal{C}_{t+1}\right)\right]-\left[\mathcal{C}_{t} \times\left\{R_{t}\right\}\right]
\end{aligned}
$$

and $\left(\left\{R_{n+t}\right\}, \bar{N}\left(\mathcal{C}_{t+1}\right)\right)_{i} \geqslant\left(\mathcal{C}_{t},\left\{R_{t}\right\}\right)_{i}$, and consequently we have

$$
\left(\mathcal{C}_{t+1}, \bar{N}\left(\mathcal{C}_{t+1}\right)\right)_{i}=\left(\mathcal{C}_{t}, \bar{N}\left(\mathcal{C}_{t}\right)\right)_{i}+\left(\left\{R_{n+t}\right\}, \bar{N}\left(\mathcal{C}_{t+1}\right)\right)_{i}-\left(\mathcal{C}_{t},\left\{R_{t}\right\}\right)_{i} \geqslant\left(\mathcal{C}_{t}, \bar{N}\left(\mathcal{C}_{t}\right)\right)_{i} .
$$

Therefore, for $1 \leqslant i \leqslant n$,

$$
(\mathcal{C}, \mathcal{D})_{i} \leqslant\left(\mathcal{C}_{s}, \bar{N}\left(\mathcal{C}_{s}\right)\right)_{i} \leqslant\left(\mathcal{C}_{s+1}, \bar{N}\left(\mathcal{C}_{s+1}\right)\right)_{i} \leqslant \cdots \leqslant\left(\mathcal{C}_{n}, \bar{N}\left(\mathcal{C}_{n}\right)\right)_{i}=a_{i}
$$


because $\mathcal{C}_{n}=\bar{N}\left(\mathcal{C}_{n}\right)$ is a maximum independent set of $\mathcal{R}$.

Now we consider the general case. Without loss of generality, assume $R_{2 n} \in \mathcal{D}$. Then $\mathcal{C} \subseteq \bar{N}(\mathcal{D}) \subseteq \bar{N}\left(\left\{R_{2 n}\right\}\right)=\left\{R_{n+1}, R_{n+2}, \ldots, R_{3 n-1}\right\}$. Suppose $\mathcal{C}=\left\{R_{i_{1}}, R_{i_{2}}, \ldots, R_{i_{s}}\right\}$, where $n+1 \leqslant i_{1}<i_{2}<\cdots<i_{s} \leqslant 3 n-1$. Noting $p \geqslant 4$, if $R_{j} \in \bar{N}\left(\left\{R_{i_{1}}\right\}\right) \cap \bar{N}\left(\left\{R_{i_{s}}\right\}\right)$, then it follows from definition that $\left|j-i_{1}\right|<n$ and $\left|j-i_{s}\right|<n$, that is, $i_{s}-n+1 \leqslant j \leqslant$ $i_{1}+n-1$. Therefore, $\bar{N}\left(\left\{R_{i_{1}}\right\}\right) \cap \bar{N}\left(\left\{R_{i_{s}}\right\}\right)=\left\{R_{i_{s}-n+1}, R_{i_{s}-n+2}, \ldots, R_{i_{1}+n-1}\right\}=\bar{N}(\mathcal{C})$. Set $\mathcal{C}^{\prime}=\left\{R_{i_{1}}, R_{i_{1}+1}, \ldots, R_{i_{s}}\right\}$ and $\mathcal{D}^{\prime}=\left\{R_{i_{s}-n+1}, R_{i_{s}-n+2}, \ldots, R_{i_{1}+n-1}\right\}$. Then, $\mathcal{C}^{\prime}=\bar{N}\left(\mathcal{D}^{\prime}\right)$, and the above argument implies that the inequality $\left(\mathcal{C}^{\prime}, \mathcal{D}^{\prime}\right)_{i} \leqslant a_{i}$ holds for each $1 \leqslant i \leqslant n$. Note that $\mathcal{C} \subseteq \mathcal{C}^{\prime}$ and $\mathcal{D} \subseteq \mathcal{D}^{\prime}$. Hence, $(\mathcal{C}, \mathcal{D})_{i} \leqslant\left(\mathcal{C}^{\prime}, \mathcal{D}^{\prime}\right)_{i} \leqslant a_{i}$.

Remark. In the above result, the condition that $p \geqslant 4$ is necessary. For example, assume $n=6$ and $p=3$, set $\mathcal{S}=\left\{R_{1}, R_{2}, R_{3}, R_{4}, R_{5}, R_{6}\right\}, \mathcal{C}=\left\{R_{6}, R_{14}\right\}$ and $\mathcal{D}=\left\{R_{1}, R_{11}\right\}$, it is easy to see that $\mathcal{S}$ is a maximum independent set of $\mathcal{R}$ and $\mathcal{C} \times \mathcal{D}$ is an independent set of $\widehat{\mathcal{R}}$, but $2=(\mathcal{S}, \mathcal{S})_{1}<(\mathcal{C}, \mathcal{D})_{1}=3 \leqslant \alpha\left(\widehat{\mathcal{R}}_{1}\right)$, and so $\sum_{i=1}^{6} \alpha\left(\widehat{\mathcal{R}}_{i}\right)>\sum_{i=1}^{6}(\mathcal{S}, \mathcal{S})_{i}=\alpha(\widehat{\mathcal{R}})$.

\section{Proof of Theorem 3}

In this section we complete the proof of Theorem 3.

Proof of Theorem 3. Take a maximum independent set $\mathcal{S}^{\prime}$ of $\mathcal{L}_{p}$ and set $\widehat{\mathcal{I}}^{\prime}=\mathcal{S}^{\prime} \times \mathcal{S}^{\prime}$. Then $\widehat{\mathcal{I}}^{\prime}$ is an independent set of $\widehat{\mathcal{L}}_{p}$ with $\left|\widehat{\mathcal{I}}^{\prime}\right|=p^{2 n-2}$. Note that $\frac{\alpha(\mathcal{R})}{|\mathcal{R}|}=\frac{\alpha\left(\mathcal{L}_{p}\right)}{\left|\mathcal{L}_{p}\right|}=\frac{\left|\mathcal{S}^{\prime}\right|}{\left|\mathcal{L}_{p}\right|}=\frac{1}{p}$. For each $\sigma \in \Gamma$, Lemma 5 implies $\left|\mathcal{S}^{\prime} \cap \sigma(\mathcal{R})\right|=\alpha(\mathcal{R})=n$, that is to say, $\left|\widehat{\mathcal{I}}^{\prime} \cap \sigma(\widehat{\mathcal{R}})\right|=n^{2}$, and so the equalities $\left|\widehat{\mathcal{I}}^{\prime} \cap \sigma(\widehat{\mathcal{R}})\right|=\alpha(\widehat{\mathcal{R}})=\sum_{i=1}^{n} \alpha\left(\widehat{\mathcal{R}}_{i}\right)$ hold by Lemma 7 . Then, it follows from Lemma 6 that $p^{2 n-2}=\left|\widehat{\mathcal{I}}^{\prime}\right|=\sum_{i=1}^{n}\left|\widehat{\mathcal{L}}_{p, i}\right| \frac{\alpha\left(\widehat{\mathcal{R}}_{i}\right)}{\left|\hat{\mathcal{R}}_{i}\right|}$. Therefore, for every independent set $\widehat{\mathcal{I}}$ of $\widehat{\mathcal{L}}_{p}$, we have $|\widehat{\mathcal{I}}| \leqslant \sum_{i=1}^{n}\left|\widehat{\mathcal{L}}_{p, i}\right| \frac{\alpha\left(\widehat{\mathcal{R}}_{i}\right)}{\left|\widehat{\mathcal{R}}_{i}\right|}=p^{2 n-2}$. Furthermore, the equality holds if and only if $|\widehat{\mathcal{I}} \cap \sigma(\widehat{\mathcal{R}})|=\alpha(\widehat{\mathcal{R}})$ for all $\sigma \in \Gamma$. Then, for each $\sigma \in \Gamma$, by Lemma 7 , $\widehat{\mathcal{I}} \cap \sigma(\widehat{\mathcal{R}})=\mathcal{S}_{\sigma} \times \mathcal{S}_{\sigma}$ for some maximum independent set $\mathcal{S}_{\sigma}$ of $\sigma(\mathcal{R})$. Set $\mathcal{S}=\cup_{\sigma \in \Gamma} \mathcal{S}_{\sigma}$. Noting that the maximality of $\widehat{\mathcal{I}}$ implies that $\widehat{\mathcal{I}}=\mathcal{C} \times \mathcal{D}$ for a pair of cross-intersecting subfamilies $\mathcal{C}$ and $\mathcal{D}$ of $\mathcal{L}_{p}$. Then we have that $\mathcal{S}$ is an independent set and $\mathcal{S} \times \mathcal{S} \subseteq \widehat{\mathcal{I}}$. On the other hand, it is easy to see that $|\mathcal{S} \cap \sigma(\mathcal{R})|=\alpha(\mathcal{R})$ holds for all $\sigma \in \Gamma$, so Lemma 5 implies $\mathcal{S}$ is a maximum independent set of $\mathcal{L}_{p}$. Then we obtain $\widehat{\mathcal{I}}=\mathcal{S} \times \mathcal{S}$ since $|\widehat{\mathcal{I}}|=p^{2 n-2}=|\mathcal{S} \times \mathcal{S}|$. This completes the proof of Theorem 3 .

\section{Acknowledgements}

The author is greatly indebted to the anonymous referees for giving useful comments and suggestions that have considerably improved the manuscript. He also thanks Jun Wang for giving many valuable suggestions. 


\section{References}

[1] M.O. Albertson and K.L. Collins, Homomorphisms of 3-chromatic graphs, Discrete Math., 54: 127-132, 1985.

[2] C. Berge, Nombres de coloration de 1'hypegraphe h-parti complet, in: Hypergraph Seminar(Columbus, Ohio 1972), volume 411 of Lecture Notes in Math., Page 13-20. Springer, 1974.

[3] C. Bey, On cross-intersecting families of sets, Graphs Combin., 21: 161-168, 2005.

[4] B. Bollobás and I. Leader, An Erdős-Ko-Rado theorem for signed sets, Comput. Math. Applic., 34: 9-13, 1997.

[5] P. Borg, Intersecting and cross-intersecting families of labeled sets, Electron. J. Combin., 15: N9, 2008.

[6] P. Borg, On t-intersecting families of signed sets and permutations, Discrete Math., 309: 3310-3317, 2009.

[7] P. Borg, Extremal $t$-intersecting sub-families of hereditary families, J. London Math. Soc., 79: 167-185, 2009.

[8] P. Borg, A short proof of a cross-intersection theorem of Hilton, Discrete Math., 309: 4750-4753, 2009.

[9] P. Borg, Cross-intersecting families of permutations, J. Combin. Theory Ser. A, 117: 483-487, 2010.

[10] P. Borg and I. Leader, Multiple cross-intersecting families of signed sets, J. Combin. Theory Ser. A, 117: 583-588, 2010.

[11] P.J. Cameron, C.Y. Ku, Intersecting families of permutations, European J. Combin., 24: 881-890, 2003.

[12] M. Deza, P. Frankl, On the maximum number of permutations with given maximal or minimal distance, J. Combin. Theory Ser. A, 22: 352-360, 1977.

[13] M. Deza and P. Frankl, Erdős-Ko-Rado theorem-22 years later, SIAMJ. Alg. Disc. Methods, 4: 419-431, 1983.

[14] D. Ellis, E. Friedgut, H. Pilpel, Intersecting families of permutations, J. Amer. Math. Soc., 24: 649-682, 2011.

[15] P. Erdős, C. Ko and R. Rado, Intersection theorems for systems of finite sets, Quart. J. Math. Oxford Ser., 2 (12): 313-318, 1961.

[16] X.B. Geng, J. Wang and H.J. Zhang, Structures of Independent Sets in Direct Products of Some Vertex-transitive Graphs, Acta Math. Sin. (Engl. Ser.), 28: 697-706, 2012.

[17] P. Frankl, R.M. Wilson, The Erdős-Ko-Rado theorem for vector spaces, J. Combin. Theory Ser. A, 43: 228-236, 1986.

[18] C. Greene and D.J. Kleitman, Proof techniques in the ordered sets, in: Studies in Combinatorics (Math. Assn. America, Washington DC, 1978), pp. 22-79. 
[19] A.J.W. Hilton, An intersection theorem for a collection of families of subsets of a finite set, J. London Math. Soc., 2: 369-384, 1977.

[20] W.N. Hsieh, Intersection theorems for systems of finite vector spaces, Discrete Math., 12: 1-16, 1975.

[21] G. O. H. Katona, A simple proof of the Erdős-Ko-Rado theorem, J. Combin. Theory Ser. B, 13:183-184, 1972.

[22] Y.S. Li, J. Wang, Erdős-Ko-Rado-Type Theorems for Colored Sets, Electron. J. Combin., 14(1), 2007.

[23] M. L. Livingston, An ordered version of the Erdős-Ko-Rado theorem, J. Combin. Theory Ser. A, 26: 162-165, 1979.

[24] M. Matsumoto, N. Tokushige, The exact bound in the Erdős-Rado-Ko theorem for cross-intersecting families, J. Combin. Theory Ser. A, 52: 90-97, 1989.

[25] L. Pyber, A new generalization of the Erdős-Rado-Ko theorem, J. Combin. Theory Ser. A, 43:85-90, 1986.

[26] N. Tokushige, On cross t-intersecting families of sets, J. Combin. Theory Ser. A, 117: 1167-1177, 2010.

[27] J. Wang, S.J. Zhang, An Erdős-Ko-Rado-type theorem in Coxeter groups, European J. Combin., 29: 1112-1115, 2008.

[28] J. Wang and H.J. Zhang, Intersecting families in a subset of boolean lattices, Electron. J. Combin., 18(P237), 2011.

[29] J. Wang and H.J. Zhang, Cross-intersecting families and primitivity of symmetric systems, J. Combin. Theory Ser. A, 118: 455-462, 2011.

[30] J. Wang and H.J. Zhang, Nontrivial independent sets of bipartite graphs and crossintersecting families, J. Combin. Theory Ser. A, 120: 129-141, 2013. 